\title{
VARIATIONAL PRINCIPLES FOR HILL'S SPHERICAL VORTEX AND NEARLY SPHERICAL VORTICES
}

\author{
YIEH-HEI WAN
}

\begin{abstract}
In this paper, vortex rings are regarded as axisymmetric motions without swirl of an incompressible inviscid fluid in space, with vorticity confined to their finite cores. The main results of this paper are $(\mathrm{H})$ Hill's spherical vortex is a "nondegenerate" local maximum of the energy function subject to a fixed impulse, among vortex rings. (N) Norbury's nearly spherical vortex is a "nondegenerate" local maximum of the energy function subject to a fixed impulse, and a fixed circulation. Estimates are established to overcome the discontinuity of vorticity distributions, and the singular behavior of Stoke's stream functions near the axis of symmetry. The spectral analysis involves the use of Legendre's functions.
\end{abstract}

1. Introduction. In this paper, we consider axisymmetric motions without swirl of an incompressible inviscid fluid in space, with zero velocity at infinity. We shall restrict ourselves to a vortex ring, in which the vorticity is confined to a bounded region (called the core) and its magnitude is equal to the distance to the axis of symmetry.

A vortex ring is said to be steady if it moves without change of shape and propagates at a constant speed along its axis of symmetry. Helminolz's vortex ring $[14,8]$ is steady and it has a solid torus with small cross section as its core. On the other hand, Hill's spherical vortex $[14, \mathbf{1 1}]$ is steady and it has a solid sphere as its core. Norbury $[\mathbf{1 8}, \mathbf{1 9}]$ found a family of steady vortex rings which connect Helmholtz's vortex ring to Hill's spherical vortex. The shape of the cores in this family changes at Hill's spherical vortex.

Variational characterizations of steady motions often enable us to draw stability results. This idea in fluid mechanics went back at least to Kelvin [13]. The variational characterizations based on stream functions for Helmholtz's rings and Hill's vortex ring have been established by Fraenkel and others $[\mathbf{1}, \mathbf{9}]$. We aim to show that one can also verify variational characterizations based on conserved quantities for Hill's spherical vortex and Norbury's nearly spherical vortex [19]. Therefore, one obtains stability results for these vortex rings within the class of axisymmetric motions.

The formulation of our variational principles was suggested by Benjamin [5] and Friedman and Turkington [10]. Indeed, the main results of this paper are as follows:

Hill's spherical vortex is a "nondegenerate" local maximum of the energy function subject to a fixed impulse, and a constraint $Z=0$.

Received by the editors November 17, 1986 and, in revised form, May 15, 1987.

1980 Mathematics Subject Classification (1985 Revision). Primary 76C05, 76E99.

Key words and phrases. Vortex rings, Hill's spectral vortex, impulse, circulations, energy-Casimir method, spherical analysis, Legendre functions.

Research partially supported by National Science Foundation under grant DMS-8501746. 

Norbury's nearly spherical vortex is a "nondegenerate" local max- imum of the energy function subject to a fixed impulse, a fixed circulation, and a constraint $Z=0$.

Notice that one has the impulse $[14,6]$ as a conserved quantity corresponding to the translation invariance nature of our problem. The constraint $Z=0$ will be defined in $\S 2$, and it is not a translation invariance. Our local maximum $\varsigma_{A}$ (in $(\mathrm{H})$ or $(\mathrm{N})$ ) is "nondegenerate" in the sense that: the negative of increments of energy at $\zeta_{A}$ bounds a positive quadratic function of the variations of vorticity within the given constraints near $\zeta_{A}$. One needs such a priori estimates in order to draw stability results from variational principles based on conserved quantities. Our results complement those of Friedman and Turkington [10] and Fraenkel and Berger [9], in which they provide the existence of steady vortex rings via variational principles.

In $[2,3]$ Arnold showed how one can establish a priori estimates in a smooth setting, and hence one can obtain a nonlinear stability result. These geometrical considerations have been exploited recently by Marsden and Weinstein [15]. They also formulated this method for general Hamiltonian systems and coined it as Casimir-energy method. For more details and applications, see Holm et al. [12]. However, one cannot apply this method to motions of vortex patches (in $R^{2}$ ), for the vorticity has discontinuity and the setting is not smooth. In [21], Wan and Pulvirenti show how to overcome this difficulty. Indeed, one can reduce this problem to a smooth one in some suitable sense. This idea is used in Tang [20] successfully to establish a variational principle and a stability result for Kirchhoff's elliptic vortex.

For axisymmetric flows, we can take a half-plane as a cross section and formulate our problem on this half-plane. This formulation is very similar to that of vortex patches. A steady vortex ring corresponds to a steady translating vortex patch. In principle, a vortex ring near a steady vortex ring allows the possibility of long thin and complicated filaments moving relatively far from the steady one, as that can happen to a vortex patch. For Hill's spherical vortex, a thin spike can grow from the rear stagnation point [16]. Thus, we like to use the method in [21] to overcome the nonsmoothness nature of our problem. Our problem has another complication due to the singular behavior (of Stokes' stream functions, etc.) near the axis of symmetry. The regularity result for Stokes' stream functions presented as Proposition 2 in the Appendix helps us to resolve this singularity.

Let us now outline the idea of proving our main results $(\mathrm{H})$ and $(\mathrm{N})$. First, by using an implicit function theorem to a suitable mapping, one makes a clever choice of a vortex $\varsigma^{*}, C^{1}$-close to the steady one $\zeta_{s}$ and satisfying the same given constraints so that one can prove $E(\varsigma)<E\left(\varsigma^{*}\right)$. Here, $E(\varsigma)$ denotes the energy of the vortex $\varsigma$. Secondly, by some lengthy computations, one can obtain a second order Taylor expansion for $E\left(\varsigma^{*}\right)$ around $\varsigma_{s}$ with a nice estimate of the remainder. Thus, $E\left(\varsigma^{*}\right)<E\left(\varsigma_{s}\right)$ follows by proving the negative definiteness of the second order term. For Hill's spherical vortex, this can be carried out by a spectral analysis which involves Legendre's functions of order 1 as eigenfunctions. For a nearly spherical vortex, it suffices to show that its second order term is approximated by that of Hill's spherical vortex in a suitable sense. Combining the above two inequalities, one has $E(\varsigma)<E\left(\varsigma_{s}\right)$. 
The precise statements of our main results in this paper are given in $\S 2$ as Theorems $(\mathrm{H})$ and $(\mathrm{N})$. The proofs of Theorems $(\mathrm{H})$ and $(\mathrm{N})$ are given in two steps in $\S \S 3$ and 4 respectively. To make these proofs readable, technical estimates and their consequences are collected and justified in the Appendices.

Finally, I would like to thank the referee for many valuable suggestions, especially the short proof of our regularity results on stream functions (i.e. Proposition 2), based on a transformation discussed in $\mathrm{Ni}$ [17] and Amick and Fraenkel [1].

2. Statement of the main results. Let $(r, \eta, z)$ be a cylindrical coordinate system on $R^{3}$. Throughout this paper, we consider the flow of an incompressible inviscid fluid with unit density in $R^{3}$ such that the velocity field is symmetric about the $z$-axis, tangent to the half-planes $\eta=$ constant, and zero at infinity. It is natural and convenient to represent such an axisymmetric flow on the meridional half-plane $(\eta=0) M \equiv\{(r, z) \mid r \geq 0,-\infty<z<\infty\}$ with measure $d \mu=2 \pi r d r d z$. At any instant, the velocity field on $M$ can be described by a (Stokes') stream function $\psi=\psi(r, z),\left(v^{r}, v^{z}\right)=\left(-\psi_{z} / r, \psi_{r} / r\right)$ and its vorticity $\omega=v_{z}^{r}-v_{r}^{z}$ given by $\omega=-(L \psi) / r$ with

$$
L=r \frac{\partial}{\partial r}\left(\frac{1}{r} \frac{\partial}{\partial r}\right)+\frac{\partial^{2}}{\partial z^{2}}
$$

We like to use a formulation in terms of a "vortex density" $\zeta$ (called vortex $\zeta$ for simplicity) on the half-plane $M$, defined as $\zeta(r, z)=\omega(r, z) / r$. We should always assume $\zeta(r, z)$ is a bounded function with compact support in $M$. For $\varsigma=-(L \psi) / r^{2}$, let us take $\psi=\int_{M} G \zeta d \mu$ as the associated stream function to $\varsigma$. Here, the Green ${ }^{1}$ function $G$ can readily be found as

$$
G\left(r, z, r^{\prime}, z^{\prime}\right)=\frac{r r^{\prime}}{8 \pi^{2}} \int_{-\pi}^{\pi} \frac{\cos \eta^{\prime} d \eta^{\prime}}{\left[\left(z-z^{\prime}\right)^{2}+r^{2}+r^{\prime 2}-2 r r^{\prime} \cos \eta^{\prime}\right]^{1 / 2}}
$$

Now, the kinetic energy $E$, the impulse $P$, and the circulation $\Gamma$ have the following expressions $[14,9,10,5]$.

$$
\begin{aligned}
& E=\frac{1}{2} \int_{M} \psi \varsigma d \mu=\frac{1}{2} \int_{M}\langle\psi, \varsigma\rangle, \\
& P=\int_{M} r^{2} \varsigma d \mu, \quad \Gamma=\int_{M} \varsigma d \mu .
\end{aligned}
$$

It is well known that $\varsigma, d \mu, E, P$, and $\Gamma$ are invariant under axisymmetric motions of an incompressible inviscid fluid governed by the vorticity equation: $\zeta_{t}+v^{r} \zeta_{r}+v^{z} \zeta_{z}=$ 0. Define also a noninvariant quantity $Z=\int_{M} z r^{2} \varsigma d \mu$, similar to those in [5, 10].

As in [18], closeness of vortices can be measured by a $L^{1}$-norm, $\|\zeta\|_{1}=\int_{M}|\zeta| d \mu$. For simplicity, we often take vortices $\zeta$ from a class $\mathcal{V}$, which consists of vortices $\zeta$ in the form $\zeta=\chi_{A}$, where $\chi_{A}$ denotes the characteristic function of some bounded set $A \subset M$. A vortex $\varsigma=\chi_{A} \in \mathcal{V}$, with $C^{1}$ boundary $\partial A$ in $M$, is called steady if the vortex $\varsigma$ propagates with some constant speed $W$ in the $z$-direction. This is equivalent to say that the relative stream function, $\tilde{\psi} \equiv \psi-\frac{1}{2} W r^{2}=$ constant on the boundary $\partial A$ of $A$ (flux constant). The Hill's spherical vortex $\varsigma_{H}$ (of radius 1 ) is a steady vortex such that $\zeta_{H}=\chi_{H}, H=\left\{(r, z) \in M \mid r^{2}+z^{2} \leq 1\right\}$ with $W_{H}=2 / 15$

\footnotetext{
${ }^{1}$ Due to different normalizations, the Green function used here and those in $[10,18]$ are all different by some factors.
} 
$[14,11]$. Denote by $\varsigma_{N}(\varepsilon)$ Norbury's steady vortex ring with speed $W(\varepsilon)$ found in $[\mathbf{1 8}, 19]$. It has the form $\zeta_{N}(\varepsilon)=\chi_{H_{\varepsilon}}, H_{\varepsilon}=\left\{(r, z) \in M \mid \tilde{\psi}_{N}(\varepsilon) \geq \gamma(\varepsilon)\right\}$ with $\tilde{\psi}_{N}(\varepsilon)=\psi_{N}(\varepsilon)-\frac{1}{2} W(\varepsilon) r^{2} . \psi_{N}(\varepsilon)=\int G_{\zeta_{N}}(\varepsilon) d \mu, \gamma(\varepsilon)>0$. $\varsigma_{N}(\varepsilon)$ approaches to Hill's spherical vortex $\varsigma_{H}, W(\varepsilon) \rightarrow W_{H}, \gamma(\varepsilon) \rightarrow 0$, and $\psi_{N}(\varepsilon) \rightarrow \psi_{H}$ pointwise as $\varepsilon \rightarrow 0+$. One can assume that $H_{\varepsilon} \subset H$, by proper scaling if necessary.

Now we are ready to state our main results.

THEOREM $(\mathrm{H})$. There exist positive constants $c_{3}, \delta$ such that the inequality $E\left(\varsigma_{H}\right)-E(\varsigma) \geq c_{3} P\left(\left|\varsigma_{H}-\varsigma\right|\right)^{2}$ holds for $\varsigma \in \mathcal{V}$ when $P(\varsigma)=P\left(\varsigma_{H}\right), Z(\varsigma)=$ $Z\left(\varsigma_{H}\right)(=0)$, and $P\left(\left|\varsigma-\varsigma_{H}\right|\right)<\delta$. Recall that $P(|\xi|)=\int r^{2}|\xi| d \mu$.

THEOREM $(\mathrm{N})$. For each small $\varepsilon>0$, there exist constants $c_{3}>0, \delta>0$ such that $E\left(\varsigma_{N}(\varepsilon)\right)-E(\varsigma) \geq c_{3}\left\|\varsigma_{N}(\varepsilon)-\varsigma\right\|_{1}^{2}$ holds for $\varsigma \in \mathcal{V}$, when $P(\varsigma)=P\left(\varsigma_{N}(\varepsilon)\right)$, $\Gamma(\varsigma)=\Gamma\left(\varsigma_{N}(\varepsilon)\right), Z(\varsigma)=Z\left(\varsigma_{N}(\varepsilon)\right)(=0)$ and $\left\|\varsigma_{N}(\varepsilon)-\varsigma\right\|_{1}<\delta$.

REMARK 1. The real numbers $R$ acts on $\mathcal{V}$ via translations along $z$-axis. The functions $E, P, \Gamma$, but not $Z$, are invariant under this action. Here, we take the normalization $Z(\varsigma)=0$ in Theorems $(\mathrm{H}),(\mathrm{N})$ to factor out translations. Otherwise, the estimates are trivially false.

REMARK 2. One expects that, in Theorem (N), $c_{3} \rightarrow 0$ as $\varepsilon \rightarrow 0$, which relates to the fact that one needs to use $P\left(\left|\zeta_{\zeta}-\varsigma_{H}\right|\right)$ instead of $\left\|{ }_{\zeta}-\varsigma_{H}\right\|_{1}$ in Theorem $(\mathrm{H})$.

REMARK 3. Formally, a steady vortex ring $\varsigma_{A}$ can be characterized as an extreme point of $E(\varsigma)$ on $\mathcal{V}$, subject to $P(\varsigma)=P\left(\varsigma_{A}\right), \Gamma(\varsigma)=\Gamma\left(\varsigma_{A}\right)$ with the propagation speed $W_{A} / 2$ and the flux constant $\gamma_{A}$ as the corresponding Lagrangian multipliers. The flux constant is zero for $\zeta_{H}$ and positive for $\zeta_{N}(\varepsilon)$. So that the circulation $\Gamma(\varsigma)$ is prescribed in Theorem $(\mathrm{N})$ but not in Theorem $(\mathrm{H})$.

Denote by $\phi_{t}(\varsigma)$ the vortex at time $t$, with initial vortex $\varsigma \cdot \Phi_{t}$ leaves $\mathcal{V}$ invariant. A steady vortex $\zeta_{A}$ is said to be stable with respect to a metric $d$ if given any $\varepsilon>0$, there exists a $\delta>0$ such that to each $\zeta \in \mathcal{V}$ with $d\left(\zeta_{,} \varsigma_{A}\right)<\delta$, and to each $t>0, d\left(\Phi_{t}(\varsigma), \Phi_{t^{*}}\left(\zeta_{A}\right)\right)<\varepsilon$ for some $t^{*}$. Intuitively, it means that $\Phi_{t}(\zeta)(t \geq 0)$ remains close to $\zeta_{A}$ modulo a translation along the $z$-axis for $\zeta$ sufficiently close $\zeta_{A}$. An analogous stability result for soliton solutions of $\mathrm{KdV}$ equation has been established by Benjamin in [4].

Using methods from Wan and Pulvirenti [21] and Tang [20], we can obtain the following stability results.

COROLlaRY $(\mathrm{H})$. Hill's spherical vortex $\varsigma_{H}$ is stable relative to the metric $d_{H}$, where

$$
d_{H}\left(\varsigma_{1}, \varsigma_{2}\right)=P\left(\left|\varsigma_{1}-\varsigma_{2}\right|\right)+\left|Z\left(\varsigma_{1}-\varsigma_{2}\right)\right| .
$$

COROLLARY $(\mathrm{N})$. For sufficiently small $\varepsilon>0$, the nearly spherical vortex $\varsigma_{N}(\varepsilon)$ is stable with respect to the metric $d_{N}$, where

$$
d_{N}\left(\varsigma_{1}, \varsigma_{2}\right)=\left\|\varsigma_{1}-\varsigma_{2}\right\|_{1}+\left|P\left(\varsigma_{1}-\varsigma_{2}\right)\right|+\left|Z\left(\varsigma_{1}-\varsigma_{2}\right)\right| .
$$

In what follows, we will establish various inequalities. Thus, for convenience, we will take $C$ as a generic constant, i.e. a constant which may have different values in different places.

3. Proof of Theorem $(\mathbf{H})$. Denote by $(\rho, \theta)$ the polar coordinates on $M=$ $\{(r, z) \mid r \geq 0\}$ with $\rho \cos \theta=z, \rho \sin \theta=r$. 
The stream function of Hill's spherical vortex $\varsigma_{H}$ is given by

$$
\psi_{H}=r^{2} \cdot \phi_{H}, \quad \text { with } \phi_{H}= \begin{cases}\frac{1}{10}\left[\frac{5}{3}-\rho^{2}\right] & \text { for } \rho<1 \\ \frac{1}{10}\left[2 / 3 \rho^{3}\right] & \text { for } \rho>1\end{cases}
$$

(See [14], also Remark 4 in Appendix.) Thus, $\phi_{H}$ has a constant value $\phi_{0}=\frac{1}{15}$ on $\rho=1$.

Step (1). Reduction to a radial case $\varsigma^{*}$ (i.e. $\varsigma^{*}=\chi_{D^{*}}, D^{*}=\{(\rho, \theta) \mid \rho \leq g(\theta)\}$ for some $C^{1}$ function $g, 0<g<1$ on $\left.[0, \pi]\right)$.

From Proposition 2 in Appendix B, to each $\zeta=\chi_{D}$, one can write $\psi$ (= $\left.\int_{D} G d \mu\right)=r^{2} \phi$ with $\phi$ a $C^{1}$ function on $M$, and $\phi$ is $C^{1}$-close to $\phi_{H}$ on $\rho \leq 2$ if $P\left(\left|\zeta-\varsigma_{H}\right|\right)$ is small. In what follows, we assume that $P\left(\left|\zeta-\varsigma_{H}\right|\right)$ is sufficiently small.

For any $\phi C^{1}$-close to $\phi_{H}$ on $\rho \leq 2, \mu=\left(\mu_{1}, \mu_{2}\right)$ close to 0 , set $\zeta_{\mu, \phi}^{*}=\chi_{D^{*}}$ with $D^{*}=\left\{(r, z) \in M \mid \rho \leq 2, \phi-\frac{1}{15}-\mu_{1} / 2-\mu_{2} z \geq 0\right\}$. Consider the map $F(\mu, \phi)=\left(P\left(\varsigma_{\mu, \phi}^{*}\right)-P\left(\varsigma_{H}\right), Z\left(\varsigma_{\mu, \phi}^{*}\right)\right)$. Computations show that

$$
\operatorname{det} D_{\mu} F\left(0, \phi_{H}\right)=\left|\begin{array}{cc}
-20 \pi / 3 & 0 \\
0 & -8 \pi / 3
\end{array}\right| \neq 0 \text {. }
$$

So for $\phi C^{1}$-close to $\phi_{H}$ on $\rho \leq 2$, there exists unique $\mu=\mu(\phi)$ close to $(0,0)$, such that $F(\mu(\phi), \phi)=0$ by an implicit function theorem. Now define $\varsigma^{*}=\varsigma_{\mu(\phi), \phi}^{*}=$ $\chi_{D^{*}}$ for each $\zeta$, with $P\left(\left|\varsigma-\varsigma_{H}\right|\right)$ sufficiently small. Observe that $P\left(\varsigma^{*}\right)=P\left(\varsigma_{H}\right)$. $Z\left(\varsigma^{*}\right)=Z\left(\varsigma_{H}\right)(=0)$, and the boundary $\partial D^{*}$ of $\varsigma^{*}$ in $M$ is $C^{1}$-close to the boundary $\rho=1$ of $\varsigma_{H}$.

Choose numbers $\phi_{1}, \phi_{2}$ close to $\phi_{0}=\frac{1}{15}, \phi_{2}<\phi_{0}<\phi_{1}$, such that $P(S)=$ $P\left(D \backslash D^{*}\right)=P\left(D^{*} \backslash D\right) P(T)$, where $\left.P^{\prime} S\right)$ means $P\left(\chi_{s}\right)$ etc., and $S=\{(r, s) \in$ $\left.M \mid \phi_{2}<\phi(r, z)<\phi_{0}\right\}, T=\left\{\phi_{0}<\phi(r, z)<\phi_{1}\right\}$. One can readily establish the following formulae (as in the proof of lemma 1 in [21]).

(i)

(ii)

$$
\begin{aligned}
P(S) & \left.=P(T)=\beta\left(\phi_{2}-\phi_{0}\right)+o\left(\mid \phi_{2}-\phi_{0}\right) \mid\right) \\
& =\beta\left(\phi_{0}-\phi_{1}\right)+o\left(\left|\phi_{0}-\phi_{1}\right|\right) \quad \text { with } \beta=-\frac{8 \pi}{15}
\end{aligned}
$$

$$
\begin{aligned}
& \int_{S} \phi r^{2} d \mu=\phi_{0}=P(S)+\frac{1}{2} \beta\left(\phi_{2}-\phi_{0}\right)^{2}+o\left(\left|\phi_{2}-\phi_{0}\right|\right), \\
& \int_{T} \phi r^{2} d \mu=\phi_{0}=P(T)-\frac{1}{2} \beta\left(\phi_{0}-\phi_{1}\right)^{2}+o\left(\left|\phi_{0}-\phi_{1}\right|\right) .
\end{aligned}
$$

(i) and (ii) imply that there exists $c_{1}>0(\approx 15 / 32 \pi)$ such that

$$
\begin{aligned}
& \int_{T} \phi r^{2} d \mu-\int_{S} \phi r^{2} d \mu \geq c_{1}(P(S)+P(T))^{2} \\
& \quad=c_{1}\left(P\left(D \backslash D^{*}\right)+P\left(D^{*} \backslash D\right)\right)^{2}=c_{1}\left[P\left(\left|\varsigma^{*}-\varsigma\right|\right)\right]^{2} .
\end{aligned}
$$

$\left\langle\varsigma^{*}-\varsigma, \psi\right\rangle \geq \int_{T} \phi r^{2} d \mu-\int_{S} \phi r^{2} d \mu$ by using the fact that $\phi>\phi_{0}$ inside $D^{*}$, $\phi<\phi_{0}$ outside $D^{*}$ and our chosen properties for $S, T$. By the above inequalities, and the fact $E\left(\varsigma^{*}-\varsigma\right) \geq 0$, one has

$$
E\left(\varsigma^{*}\right)-E(\varsigma)=\left\langle\varsigma^{*}-\varsigma, \psi\right\rangle+E\left(\varsigma^{*}-\varsigma\right) \geq c_{1}\left[\int_{M} r^{2}\left|\varsigma^{*}-\varsigma\right| d \mu\right]^{2}
$$


Thus it remains to show that

$$
E\left(\varsigma_{H}\right)-E\left(\varsigma^{*}\right) \geq c_{2} P\left(\left.\right|_{\varsigma_{H}}-\varsigma^{*} \mid\right)^{2} \text { for some constant } c_{2}>0 \text {. }
$$

Indeed,

$$
\begin{aligned}
E\left(\varsigma_{H}\right)-E(\varsigma) & =\left(E\left(\varsigma_{H}\right)-E\left(\varsigma^{*}\right)\right)+\left(E\left(\varsigma^{*}\right)-E(\varsigma)\right) \\
& \geq c_{2} P\left(\left|\varsigma_{H}-\varsigma^{*}\right|\right)^{2}+c_{1} P\left(\left|\varsigma^{*}-\varsigma\right|\right)^{2} \\
& \geq c_{3} P\left(\left|\zeta_{H}-\varsigma\right|\right)^{2} \quad \text { with } c_{3}=\frac{1}{2} \min \left(c_{1}, c_{2}\right) .
\end{aligned}
$$

Step (2). Second order analysis. To each $C^{1}$ function $g$ on $[0, \pi], 0<g<1$, define a vortex

$$
\chi_{g}= \begin{cases}1 & \text { if } \rho \leq g(\theta) \\ 0 & \text { otherwise }\end{cases}
$$

(Thus, $\varsigma_{H}=\chi_{1}$.) Set $\psi_{g}=\int G \chi_{g}, J=d \mu / d x=2 \pi r \rho(d x=d r d z)$, and $J_{0}=$ $\left.J\right|_{\rho=1}=2 \pi \sin \theta$. To functions $k, k^{\prime}$ on $[0, \pi]$, let $\left\langle\left\langle k, k^{\prime}\right\rangle\right\rangle=\int_{0}^{\pi} \sin \theta k(\theta) k^{\prime}(\theta) d \theta$, and $\|k\|_{2}^{2}=\langle\langle k, k\rangle\rangle$.

The second order Taylor expansion of $E\left(\chi_{1+h}\right)$ near $\chi_{1}$ is given by

$$
\begin{aligned}
& E\left(\chi_{1+h}\right)-E\left(\chi_{1}\right)=\left.\frac{1}{2} \int_{0}^{\pi} \frac{\partial \tilde{\psi}_{H}}{\partial \rho}\right|_{\rho=1} J_{0} h^{2} d \theta \\
& +\frac{1}{2} \int_{0}^{\pi} \int_{0}^{\pi} G_{0} J_{0} J_{0}^{\prime} h h^{\prime} d \theta d \theta^{\prime}+o\left(\left\|J_{0} h\right\|_{2}^{2}\right)
\end{aligned}
$$

where, $J_{0}^{\prime}=J_{0}\left(\theta^{\prime}\right), h^{\prime}=h\left(\theta^{\prime}\right), G_{0}=G\left(1, \theta ; 1, \theta^{\prime}\right)$. We sketch the proof here, for it is a modification of a proof presented in Proposition 2, [21].

$$
E\left(\chi_{1+h}\right)-E\left(\chi_{1}\right)=\left\langle\chi_{1+h}-\chi_{1}, \psi_{1}\right\rangle+\frac{1}{2}\left\langle\chi_{1+h}-\chi_{1}, \psi_{1+h}-\psi_{1}\right\rangle .
$$

Using the fact that $\psi_{1}=r^{2} \cdot \phi_{H}$, one can have

$$
\left(\psi_{1}\right) J=\left(\psi_{1} \cdot J\right)(1, \theta)+h(\theta)\left[\psi_{1} \frac{\partial J}{\partial \rho}+J \frac{\partial \psi_{1}}{\partial \rho}\right](1, \theta)+o\left(\sin ^{2} \theta|h(\theta)|\right) .
$$

Thus,

$$
\begin{aligned}
\left\langle\chi_{1+h}-\chi_{1}, \psi_{1}\right\rangle= & \left.\int_{0}^{\pi} \psi_{1}\right|_{\rho=1} J_{0} h d \theta+\left.\frac{1}{2} \int_{0}^{\pi}\left[\psi_{1} \frac{\partial J}{\partial \rho}+J \frac{\partial \psi_{1}}{\partial \rho}\right]\right|_{\rho=1} h^{2} d \theta \\
& +o\left(\left\|J_{0} h\right\|_{2}^{2}\right)
\end{aligned}
$$

From the uniform boundedness of the term $\int_{0}^{\pi}\left(G / \sqrt{\sin \theta} \sqrt{\sin \theta^{\prime}}\right)^{2} d \theta^{\prime}$, one can establish

$$
\left(\psi_{1+h}-\psi_{1}\right)\left(\rho^{\prime}, \theta^{\prime}\right)=\int_{0}^{\pi} G_{0} J_{0}(\theta) h(\theta) d \theta+o\left(\sqrt{\sin \theta^{\prime}}\left\|J_{0} h\right\|_{2}\right)
$$

and

$$
\begin{aligned}
& \int_{0}^{\pi} d \theta^{\prime} \int_{1}^{1+h\left(\theta^{\prime}\right)} J^{\prime} d \rho^{\prime}\left\{\int_{0}^{\pi} G_{0} J_{0} h d \theta\right\} \\
& =\frac{1}{2} \int_{0}^{\pi} \int_{0}^{\pi} G_{0} J_{0} J_{0}^{\prime} h h^{\prime} d \theta d \theta^{\prime}+o\left(\left\|J_{0} h\right\|_{2}^{2}\right)
\end{aligned}
$$


Equations (3.4) and (3.5) imply

$$
\left\langle\chi_{1+h}-\chi_{1}, \psi_{1+h}-\psi_{1}\right\rangle=\int_{0}^{\pi} \int_{0}^{\pi} G_{0} J_{0} J_{0}^{\prime} h h^{\prime} d \theta d \theta^{\prime}+o\left(\left\|J_{0} h\right\|_{2}^{2}\right) .
$$

Notice also,

$$
\begin{aligned}
0 & =P\left(\chi_{1+h}\right)-P\left(\chi_{1}\right) \\
& =\left.\int_{0}^{\pi} r^{2}\right|_{\rho=1} J_{0} h d \theta+\left.\frac{1}{2} \int_{0}^{\pi}\left[r^{2} \frac{\partial J}{\partial \rho}+J \frac{\partial r^{2}}{\partial \rho}\right]\right|_{\rho=1} h^{2} d \theta+o\left(\left\|J_{0} h_{2}^{2}\right\|\right) .
\end{aligned}
$$

The second order Taylor expansion formula (3.1) follows from equations (3.2), (3.3), (3.6), (3.7) and the relative stream function $\tilde{\psi}_{H} \equiv \psi_{1}-\frac{1}{2} W_{H} r^{2}=0$ on $\rho=1$.

Introducing the function $k=J_{0} h$, and using $\partial \tilde{\psi}_{H} / \partial \rho=-\frac{1}{5} \sin ^{2} \theta$,

$$
E\left(\chi_{1+h}\right)-E\left(\chi_{1}\right)=\frac{1}{2}\langle\langle k, T k\rangle\rangle+o\left(\|k\|_{2}^{2}\right)
$$

where

$$
T k=-\frac{1}{10 \pi} k+K k \quad \text { and } \quad K k(\theta)=\frac{1}{\sin \theta} \int_{0}^{\pi} G_{0} k\left(\theta^{\prime}\right) d \theta^{\prime},
$$

a bounded selfadjoint linear operator.

By Proposition 1 in Appendix A, the selfadjoint operator $T$ has eigenvalues $-1 / 10 \pi+1 / 2 \pi(2 n+1), n=1,2, \ldots$, with eigenfunctions $P_{n}^{1}(\cos \theta)$. Here, $\left\{P_{n}^{1}\right\}$ denotes the Legendre functions of order 1 .

Let $k=k_{t}+k_{n}$ be a $L^{2}$-orthogonal eigenspace decomposition of $T$, with $\left\{k_{n}\right\}$ the space spanned by $P_{1}^{1}=\sin \theta, P_{2}^{1}=3 \sin \theta \cos \theta$. T has eigenvalues $\leq-1 / 60 \pi$, when restricting to the eigenspace $\left\{k_{n}\right\}$. Therefore, there exists $\eta>0$ (small) such that

$$
\langle\langle k, T k\rangle\rangle \leq-(1 / 70 \pi)\|k\|_{2}^{2}, \quad \text { if }\left\|k_{n}\right\|_{2}<\eta\|k\|_{2} .
$$

$P\left(\chi_{1+h}\right)=P\left(\chi_{1}\right), Z\left(\chi_{1+h}\right)=Z\left(\chi_{1}\right)$ imply $\left\|k_{n}\right\|_{2}=O\left(\|k\|_{2}^{2}\right)$ (cf. (3.7)). Thus, equations (3.9) and (3.8) give

$$
E\left(\chi_{1+h}\right)-E\left(\chi_{1}\right) \leq-\frac{1}{80 \pi}\left\|J_{0} h\right\|_{2}^{2}
$$

Finally,

$$
\begin{aligned}
P\left(\left|\chi_{1+h}-\chi_{1}\right|\right) & =\int_{0}^{\pi}\left|\int_{1}^{1+h} 2 \pi \rho \sin \theta \cdot \rho^{2} \sin ^{2} \theta \cdot \rho d \rho\right| d \theta \\
& \leq C \int_{0}^{\pi} \sin ^{3 / 2} \theta\left|\sqrt{\sin \theta} J_{0} h\right| d \theta \quad(C>0) \\
& \leq C\left\|J_{0} h\right\|_{2} \quad \text { (by Schwarz inequality) }
\end{aligned}
$$

From equations (3.10) and (3.11) we obtain the required inequality in Step (2), $E\left(\varsigma_{H}\right)-E\left(\varsigma^{*}\right) \geq c_{2} P\left(\left|\zeta_{H}-\varsigma^{*}\right|\right)^{2}$ for some constant $c_{2}>0$.

Clearly, combining Step (1) and Step (2), we establish the desired inequality, and thus, we complete the proof of Theorem $(\mathrm{H})$. 
4. Proof of Theorem (N). We find it is convenient to use the polar-like coordinates $\left(-\tilde{\psi}_{N}(\varepsilon), \alpha\right)$ on $M$. Recall that $\tilde{\psi}_{N}(\varepsilon)$ stands for the relative stream function of Norbury's nearly spherical vortex $\varsigma_{N}(\varepsilon)=\chi_{H_{\varepsilon}}$, and $\alpha$ is the angle between the ray $\overrightarrow{X_{0} X}$ and $\overrightarrow{X_{0} X_{1}}$, where $X_{0}=\left(\frac{1}{2}, 0\right), X_{1}=\left(\frac{1}{2}, 1\right)$ and $X=(r, z)$.

In what follows, $\varepsilon>0$ will be fixed and small (to be determined later). Also, for such a $\varepsilon>0, \varsigma$ is sufficiently $L^{1}$-close to $\varsigma_{N}(\varepsilon)$.

Set $I_{\varepsilon}=d \mu /\left.d\left(-\tilde{\psi}_{N}(\varepsilon), \alpha\right)\right|_{\partial H_{\varepsilon}}$, and claim that

$$
0<I_{\varepsilon} \leq 80 \pi / r \text { for }(r, z) \in \partial H_{\varepsilon} .
$$

Indeed,

$$
\begin{aligned}
\frac{2 \pi}{I_{\varepsilon}} & =\frac{1}{r} \frac{\partial\left(\tilde{\psi}_{N}(\varepsilon), \alpha\right)}{\partial(r, z)} \\
& =\frac{1}{10}\left|\begin{array}{cc}
2-4 r^{2}-2 z^{2}+2 \phi_{\varepsilon}+r\left(\phi_{\varepsilon}\right)_{r} & -2 r z+r\left(\phi_{\varepsilon}\right)_{z} \\
\frac{\partial \alpha}{\partial r} & \frac{\partial \alpha}{\partial z}
\end{array}\right|
\end{aligned}
$$

Thus

$$
\frac{2 \pi \rho_{0}}{I_{\varepsilon}}=\frac{1}{5}\left[\frac{10 \gamma_{\varepsilon}}{r^{2}}\left(\frac{1}{2}-2 r\right)+r\left(1-\frac{r}{2}\right)+\frac{1}{2} r\left(\phi_{\varepsilon}\right)_{r}\left(\frac{1}{2}-r\right)-\frac{1}{2} r z\left(\phi_{\varepsilon}\right)_{z}\right] .
$$

Here, we represent $\partial H_{\varepsilon}$ as

$$
\tilde{\psi}_{N}(\varepsilon)=\frac{r^{2}}{10}\left[\left(1-r^{2}-z^{2}\right)+\phi_{\varepsilon}\right]=\gamma_{\varepsilon} \quad(=\gamma(\varepsilon)>0)
$$

with $\gamma_{\varepsilon}, \phi_{\varepsilon},\left(\phi_{\varepsilon}\right)_{r},\left(\phi_{\varepsilon}\right)_{z} \rightarrow 0$ as $\varepsilon \rightarrow 0$. Let $\rho_{0}=$ distance from $X_{0}$ to $(r, z)\left(\in \partial H_{\varepsilon}\right)$ (so $\left.\rho_{0} \leq 2\right)$. Using $\left(10 \gamma_{\varepsilon} / r^{2}\right)\left(\frac{1}{2}-2 r\right) \geq-r / 10$, one gets

$$
\frac{2 \pi \rho_{0}}{I_{\varepsilon}} \geq \frac{r}{5}\left[1-\frac{r}{2}-\frac{r}{8}\right] \geq \frac{r}{5} \cdot \frac{1}{4} \quad(\text { for } r \leq 6 / 5) .
$$

Thus, $0<I_{\varepsilon} \leq(40 \pi) \rho_{0} / r \leq 80 \pi / r$.

From equation (4.2),

$$
\frac{1}{I_{\varepsilon}} \rightarrow \frac{1}{I_{0}} \equiv \begin{cases}\left.\frac{1}{10 \pi} \sin \theta \frac{d \alpha}{d \theta}\right|_{\rho=1} & \text { on } \rho=1, r \geq 0 \\ \left.\frac{1}{10 \pi}\left(1-z^{2}\right) \frac{d \alpha}{d z}\right|_{r=0} & \text { on } r=0,|z| \leq 1\end{cases}
$$

pointwise, as $\varepsilon \rightarrow 0$.

Step (1). Reduction to a radial case $\varsigma^{*}$. For any $\psi C^{1}$-close to $\psi_{N}(\varepsilon)$ on $\rho \leq 2$, $\mu=\left(\mu_{1}, \mu_{2}, \mu_{3}\right)$ close to 0 , set $\varsigma_{\mu, \psi}^{*}=\chi_{D^{*}}$ with

$$
D^{*}=\left\{(r, z) \in M \mid \rho \leq 2, \psi-\frac{1}{2} W_{N}(\varepsilon) r^{2}-\frac{1}{2} \mu_{1} r^{2}-\mu_{2} z r^{2}-\mu_{3} \geq 0\right\} .
$$

Consider the map

$$
F(\mu, \psi)=\left(P\left(\varsigma_{\mu, \psi}^{*}\right)-P\left(\varsigma_{N}(\varepsilon)\right), Z\left(\varsigma_{\mu, \psi}^{*}\right), \Gamma\left(\varsigma_{\mu, \psi}^{*}\right)-\Gamma\left(\varsigma_{N}(\varepsilon)\right)\right) .
$$

Computations show

$$
D_{\mu} F\left(0, \psi_{N}(\varepsilon)\right)=\left|\begin{array}{lll}
-\int_{0}^{2 \pi} r^{2} I_{\varepsilon}\left(\frac{r^{2}}{2}\right) d \alpha & -\int_{0}^{2 \pi} r^{2} I_{\varepsilon}\left(z r^{2}\right) d \alpha & -\int_{0}^{2 \pi} r^{2} I_{\varepsilon} d \alpha \\
-\int_{0}^{\pi} r^{2} z I_{\varepsilon}\left(\frac{r^{2}}{2}\right) d \alpha & -\int_{0}^{2 \pi} r^{2} z I_{\varepsilon}\left(r^{2} z\right) d \alpha & -\int_{0}^{2 \pi} r^{2} z I_{\varepsilon} d \alpha \\
-\int_{0}^{2 \pi} I_{\varepsilon}\left(\frac{r^{2}}{2}\right) d \alpha & -\int_{0}^{2 \pi} I_{\varepsilon}\left(r^{2} z\right) d \alpha & -\int_{0}^{2 \pi} I_{\varepsilon} d \alpha
\end{array}\right| .
$$


Using the estimate $I_{\varepsilon} \leq 80 \pi / r$, one can obtain

$$
D_{\mu} F\left(0, \psi_{N}(\varepsilon)\right) \rightarrow\left|\begin{array}{ccc}
-20 \pi / 3 & 0 & -20 \pi \\
0 & -8 \pi / 3 & 0 \\
-10 \pi & 0 & -\infty
\end{array}\right| \text { as } \varepsilon \rightarrow 0
$$

Thus, $\operatorname{det} D_{\mu} F\left(0, \psi_{N}(\varepsilon)\right) \neq 0$, for $\varepsilon$ small. So for $\psi C^{1}$-close to $\psi_{N}(\varepsilon)$ on $\rho \leq 2$. There exists a unique $\mu=\mu(\psi)$ close to 0 such that $F(\mu(\psi), \psi)=0$. Now, set $\varsigma^{*}=\varsigma_{\mu(\psi), \psi}^{*}=\chi_{D^{*}}$. Observe that, for this $\varsigma^{*} P\left(\varsigma^{*}\right)=P\left(\varsigma_{N}(\varepsilon)\right), \Gamma\left(\varsigma^{*}\right)=\Gamma\left(\varsigma_{N}(\varepsilon)\right)$, $Z\left(\varsigma^{*}\right)=Z\left(\varsigma_{N}(\varepsilon)\right)(=0)$, and the boundary $\partial D^{*}$ of $\varsigma^{*}$ is $C^{1}$-close to boundary $\partial H_{\varepsilon}$ of $\varsigma_{N}(\varepsilon)\left(=\chi_{H_{\varepsilon}}\right)$.

Since $\tilde{\psi}(\varepsilon) \leq 0$ is a neighborhood of $H_{\varepsilon}$ in the half-plane $M$. The arguments in $[21]$ can be carried through easily. Thus, one gets $\left\langle\varsigma^{*}-\zeta, \psi\right\rangle \geq c_{1}\left\|\varsigma^{*}-\varsigma\right\|_{1}^{2}$ for some constant $c_{1}>0$ and it remains to show that

$$
E\left(\varsigma_{N}(\varepsilon)\right)-E\left(\varsigma^{*}\right) \geq c_{2}\left\|\varsigma_{N}(\varepsilon)-\varsigma^{*}\right\|_{1}^{2} \text { for some constant } c_{2}>0 .
$$

Step (2). Second order analysis. To each $C^{1}$ function $g=g(\alpha)$ on $[0,2 \pi]$ $(-2 \gamma(\varepsilon)<g<0)$, define a vortex

$$
\chi_{g}:= \begin{cases}1 & \text { if }-\tilde{\psi}_{N}(\varepsilon) \leq g(\alpha), \\ 0 & \text { otherwise. }\end{cases}
$$

(Thus, $\varsigma_{N}(\varepsilon)=\chi_{-\gamma(\varepsilon)}$.) To functions $k=k(\alpha), k^{\prime}=k^{\prime}(\alpha)$ on $[0,2 \pi]$, let $\left\langle k, k^{\prime}\right\rangle=$ $\int_{0}^{2 \pi} k(\alpha) k^{\prime}(\alpha) d \alpha$, and $|k|_{2}^{2}=\langle k, k\rangle$. By arguments as in [21], we obtain the second order Taylor expansion of $E\left(\chi_{-\gamma(\varepsilon)+h}\right)$ near $\chi_{-\gamma(\varepsilon)}$ :

$$
\begin{aligned}
E(\chi-\gamma(\varepsilon)+h)-E\left(\chi_{-\gamma(\varepsilon)}\right)= & \frac{1}{2} \int_{0}^{2 \pi} I_{\varepsilon} h^{2} d \alpha+\frac{1}{2} \int_{0}^{2 \pi} \int_{0}^{2 \pi} G_{\varepsilon} I_{\varepsilon} I_{\varepsilon^{\prime}} h h^{\prime} d \alpha d \alpha^{\prime} \\
& +o\left(\left|I_{\varepsilon} h\right|_{2}^{2}\right) .
\end{aligned}
$$

where $G_{\varepsilon}=\left.G\right|_{\partial H_{\varepsilon}}$. This formula is easier to get than the analogue formula (3.1) for $\varsigma_{H}$, because one needs to worry about the weight $\sin \theta$ in estimating the remainder term in (3.1).

Introducing $\sqrt{I_{\varepsilon}} h=k$, and noticing $\left|I_{\varepsilon} h\right|_{2} \leq C(\varepsilon)|k|_{2}$ (with $C(\varepsilon) \rightarrow \infty$, as $\varepsilon \searrow 0)$,

$$
E(\chi-\gamma(\varepsilon)+h)-E(\chi-\gamma(\varepsilon))=\frac{1}{2}\left\langle k, T_{\varepsilon} k\right\rangle+o\left(|k|_{2}^{2}\right)
$$

where $T_{\varepsilon} k=-k+K_{\varepsilon} k$, and

$$
K_{\varepsilon} k=\int_{0}^{2 \pi} K_{\varepsilon} k^{\prime} d \alpha^{\prime}, \quad \text { with } \mathcal{K}_{\varepsilon}=G_{0} \sqrt{1_{\varepsilon}} \sqrt{I_{\varepsilon}^{\prime}} .
$$

Define

$$
K_{0} k=\int_{0}^{2 \pi} K_{0} k^{\prime} d \alpha^{\prime}, \quad \text { with } K_{0}=G_{0} \sqrt{I_{0}} \sqrt{I_{0}^{\prime}} .
$$

One can establish that

$$
\int_{0}^{2 \pi} \mathcal{K}_{0}^{4}\left(\alpha, \alpha^{\prime}\right) d \alpha^{\prime}, \quad \int_{0}^{2 \pi} \mathcal{K}_{\varepsilon}^{4}\left(\alpha, \alpha^{\prime}\right) d \alpha^{\prime} \leq C
$$


for some constant $C>0$ (i.e. independent of $\alpha$ ). We illustrate only the proof of the more complicated case: $\int_{0}^{\pi} K_{\varepsilon}^{4} d \alpha^{\prime} \leq C$. Indeed by $I_{\varepsilon} \leq 80 \pi / r$ and Lemma 1 (in Appendix B),

$$
\begin{aligned}
\int K_{\varepsilon}^{4} d \alpha \leq & C \int_{s \leq r / 2, r \leq r^{\prime}} \ln ^{4}\left(\frac{r}{s}\right) \frac{d \alpha^{\prime}}{d s} d s+C \int_{s \leq r / 2, r^{\prime}<r} \ln ^{4}\left(\frac{r}{s}\right) \frac{d \alpha^{\prime}}{d(-s)} d(-s) \\
& +C \int_{s \geq r / 2}\left(\frac{r^{3 / 2} r^{\prime 3 / 2}}{s^{3}}\right)^{4} d \alpha^{\prime} .
\end{aligned}
$$

From a geometric consideration about $\partial H_{\varepsilon}$ as shown in Appendix $\mathrm{C}, d \alpha^{\prime} / d s$, $d \alpha^{\prime} / d(-s) \leq C$ (i.e. Corollary 2). Observe also

$$
\left(\frac{r r^{\prime}}{s^{2}}\right)^{6} \leq\left(\frac{r}{s} \frac{r+s}{s}\right)^{6} \leq[2(2+1)]^{6} \quad\left(\text { for } r^{\prime} \leq r+s\right) .
$$

Hence, we obtain

$$
\int K_{\varepsilon}^{4} d \alpha^{\prime} \leq C
$$

As a consequence of these estimates,

$$
\iint\left|\mathcal{K}_{\varepsilon}-\mathcal{K}_{0}\right|^{2} d \alpha d \alpha^{\prime} \rightarrow 0 \quad \text { as } \varepsilon \rightarrow 0
$$

and $K_{\varepsilon}$ is $L^{2}$-close to $K_{0}$ as linear transformations.

It is natural to regard $k(\alpha)$ as a function defined on $\rho=1, r \geq 0$ and $r=0,|z| \leq$ 1. Identify $k(\alpha)$ as $\left(k^{1}(\theta), k^{2}(z)\right)$ as a $L^{2}$-space, where $k^{1}=\left.k\right|_{\rho=1, r \geq 0} \sqrt{d \alpha / d \theta_{\rho=1}}$, $k^{2}=\left.k\right|_{r=0,|z| \leq 1} \sqrt{d \alpha / d z_{r=0}}$. Thus, $K_{0}\left(k^{1}, k^{2}\right)=\left(K k^{1}, 0\right)$ with

$$
K k^{1}(\theta)=10 \pi \int_{0}^{\pi} \frac{G_{0}}{\sqrt{\sin \theta} \sqrt{\sin \theta^{\prime}}} k^{1}\left(\theta^{\prime}\right) d \theta^{\prime} .
$$

So the operator $T=-I+K_{0}$ has eigenvalues $-1,-1+5 /(2 n+1)$ with eigenfunctions $\left(0, k^{2}(z)\right),\left(\sqrt{\sin \theta} P_{n}^{1}(\cos \theta), 0\right), n=1,2, \ldots$

Let $k=k_{t}+k_{n}$ be a $L^{2}$-orthogonal eigenspace decomposition with $\left\{k_{n}\right\}$ the subspace spanned by $\left(\sqrt{\sin \theta} P_{1}^{1}, 0\right)$ and $\left(\sqrt{\sin \theta} P_{2}^{1}, 0\right)$. The operator $-I+K_{0}$ has eigenvalues $\leq-\frac{1}{6}$, when restricting to the eigenspace $\left\{k_{t}\right\}$. Hence, there exists $\eta>0$ (small) such that

$$
\langle k, T k\rangle \leq-\frac{1}{7}|k|_{2}^{2} \quad \text { if }\left|k_{n}\right|_{2}<\eta|k|_{2} .
$$

For $T_{\varepsilon}=-I+K_{\varepsilon}$ if $L^{2}$-close to $T=-I+K_{0}$, we have

$$
\left\langle k, T_{\varepsilon} k\right\rangle \leq-\frac{1}{8}|k|_{2}^{2} \quad \text { if }\left|k_{n}\right|_{2}<\eta|k|_{2} .
$$

From the expansion,

$$
\begin{aligned}
0 & =P\left(\chi_{-\gamma(\varepsilon)+h}\right)-P\left(\chi_{-\gamma(\varepsilon)}\right) \\
& =\left.\int_{0}^{2 \pi} r^{2}\right|_{\partial H_{\varepsilon}} I_{\varepsilon} h d \alpha+\frac{1}{2} \int_{0}^{2 \pi} \frac{\partial r^{2} I_{\varepsilon}}{\partial\left(-\tilde{\psi}_{N}(\varepsilon)\right)} h^{2} d \alpha+o\left(\left|I_{\varepsilon} h\right|_{2}^{2}\right)
\end{aligned}
$$

one gets

$$
\left\langle e_{1}(\varepsilon), k\right\rangle \leq C(\varepsilon)|k|_{2}^{2}, \quad \text { with } e_{1}(\varepsilon)=\left.r^{2}\right|_{\partial H_{\varepsilon}} \sqrt{I_{\varepsilon}} .
$$


Similarly, from the expansion of $Z\left(\chi_{-\gamma(\varepsilon)+h}\right)\left(=Z\left(\chi_{-\gamma(\varepsilon)}\right)\right)$ one has

$$
\left\langle e_{2}(\varepsilon), k\right\rangle \leq C(\varepsilon)|k|_{2}^{2}, \quad \text { with } e_{2}(\varepsilon)=\left.z r^{2}\right|_{\partial H_{\varepsilon}} \sqrt{I_{\varepsilon}} .
$$

Now, $e_{1}(\varepsilon) \rightarrow e_{1} \equiv \sqrt{10 \pi}\left(\sqrt{\sin \theta} P_{1}^{1}, 0\right)$ in $L^{2}$-norm as $\varepsilon \rightarrow 0$, and $e_{2}(\varepsilon) \rightarrow e_{2} \equiv$ $(\sqrt{10 \pi} / 3)\left(\sqrt{\sin \theta} P_{2}^{1}, 0\right)$ in $L^{2}$-norm as $\varepsilon \rightarrow 0$. (Recall that $I_{\varepsilon} \leq 80 \pi / r$ from equation (4.1).)

$$
\begin{aligned}
\left|k_{n}\right| & =\left|\frac{\left\langle e_{1}, k\right\rangle e_{1}}{\left|e_{1}\right|_{2}^{2}}+\frac{\left\langle e_{2}, k\right\rangle e_{2}}{\left|e_{2}\right|_{2}^{2}}\right| \leq C\left[\left|\left\langle e_{1}, k\right\rangle\right|+\left|\left\langle e_{2}, k\right\rangle\right|\right] \\
& \leq C\left[\left\langle\mid e_{1}-e_{1}(\varepsilon), k\right\rangle|+|\left\langle e_{1}(\varepsilon), k\right\rangle|+|\left\langle e_{2}-e_{2}(\varepsilon), k\right\rangle|+|\left\langle e_{2}(\varepsilon), k\right\rangle \mid\right] \\
& =C(\varepsilon)|k|_{2}^{2}+\frac{\eta}{2}|k|_{2} \quad \text { for some small but fixed } \varepsilon>0(\text { by }(4.6),(4.7)) \\
& \leq \eta|k|_{2} \text { for }|k| \text { small. }
\end{aligned}
$$

Equations (4.4) and (4.5) give

$$
E\left(\chi_{-\gamma(\varepsilon)+h}\right)-E\left(\chi_{-\gamma(\varepsilon)}\right) \leq-\frac{1}{17}|k|_{2}^{2}
$$

Finally,

$$
\begin{aligned}
\left|\chi_{-\gamma(\varepsilon)+h}-\chi_{-\gamma(\varepsilon)}\right|_{1} & =\int_{0}^{2 \pi}\left|\int_{-\gamma(\varepsilon)}^{-\gamma(\varepsilon)+h} I_{\varepsilon} d\left(-\tilde{\psi}_{N}(\varepsilon)\right)\right| d \alpha \\
& \leq C\left|I_{\varepsilon} h\right|_{2} \text { for }|h|_{2} \text { small. }
\end{aligned}
$$

Thus, equations (4.9) and (4.10) imply the required inequality,

$$
E\left(\chi_{-\gamma(\varepsilon)+h}\right)-E\left(\chi_{-\gamma(\varepsilon)}\right) \geq-c_{2}\left|\chi_{-\gamma(\varepsilon)+h}-\chi_{-\gamma(\varepsilon)}\right|_{1}^{2} .
$$

By combining Steps (1) and (2), we obtain the desired inequality and we complete the proof of Theorem $(\mathrm{N})$.

\section{APPENDICES}

(A) Some integrals involving $G$.

Consider the equation $-L \psi / r^{2}=\varsigma$ in polar coordinates $(\rho, \theta)$,

$$
L \psi=\psi_{\rho \rho}-\frac{\cos \theta}{\rho^{2} \sin \theta} \psi_{\theta}+\frac{\psi_{\theta \theta}}{\rho^{2}} .
$$

Let $\phi_{n}$ be a $C^{1}$ function defined by

$$
\phi_{n}= \begin{cases}-\rho^{n+1}+c_{1} \rho^{n-1} & \text { for } \rho \leq a, n=1,2, \ldots, \\ c_{2} \rho^{-n-2} & \text { for } \rho>a,\end{cases}
$$

where $c_{1}=[(2 n+3) /(2 n+1)] a^{2}, c_{2}=[2 /(2 n+1)] a^{2 n+3}$. Set

$$
\xi_{n}= \begin{cases}(4 n+6) \rho^{n-1} & \text { for } \rho \leq a, \\ 0 & \text { for } \rho>a .\end{cases}
$$

PROPOSITION 1. (a)

$$
\rho^{2} \sin \theta \phi_{n} \frac{d P_{n}}{d \tau}=\int_{0}^{a} \int_{0}^{\pi} G \xi_{n} \frac{d P_{n}}{d \tau} d \mu, \quad \tau=\cos \theta .
$$


(b)

$$
\frac{1}{2 \pi(2 n+1)} \sin ^{2} \theta \frac{d P_{n}}{d \tau}=\int_{0}^{\pi} G \frac{d P_{n}}{d \tau} \sin \theta^{\prime} d \theta^{\prime}
$$

Here, $P_{n}$ stands for the Legendre polynomial (indeed, $\left.P_{n}^{1}(\tau)=\left(1-\tau^{2}\right)^{1 / 2} d P_{n} / d \tau\right)$.

Proof. Let $\psi_{n}=\rho^{2} \sin ^{2} \theta \phi_{n} d P_{n} / d \tau$ and $\zeta_{n}=\xi_{n} d P_{n} / d \tau$. (a) follows by checking $\psi_{n} \in C^{1}, \psi_{n} \rightarrow 0$ as $\rho \rightarrow \infty$, and $-L \psi_{n} / r^{2}=\zeta_{n}$. To obtain (b) we differentiate (a) with respect to $a$ at $\rho=a=1$.

Remark 4. (1) Putting $n=1$ in (a), it gives the stream function of Hill's spherical vortex.

(2) (b) implies that $G$ has a series expansion

$$
G=\frac{\sin \theta \sin \theta^{\prime}}{4 \pi} \sum \frac{P_{n}^{1}(\cos \theta) P_{n}^{1}\left(\cos \theta^{\prime}\right)}{n(n+1)} .
$$

This fact can be found in $[\mathbf{1 8}$, p. 267].

(B) Regularity results for $-L \psi / r^{2}=\varsigma$.

(1) For $\psi=\int G_{\zeta} d \mu$, estimates about kernel $G$ will provide information about $\psi$. Let us state Lemma 3.3 in [10] as

LEMMA 1. (i) $(0 \leq) G \leq C r \ln (r / s)$ for $s \leq r / 2$.

(ii) $(0 \leq) G \leq C r^{2} r^{\prime 2} / s^{3}$ for $s \geq r / 2$, where $G=G\left(r, z, r^{\prime}, z^{\prime}\right), s^{2}=\left(r-r^{\prime}\right)^{2}+\left(z-z^{\prime}\right)^{2}$.

Arguing as in Lemma 3.4 [10], one can establish

COROLlaRY 1. For $\varsigma \in \mathcal{V}$ with $\int \varsigma d \mu, \int r^{2} \varsigma d \mu \leq N$,

$$
(0<) \psi(r, z) \leq C(N+1) \min \left\{r, r^{-1 / 2}\right\}
$$

Hence, $\psi$ is bounded and $\psi \rightarrow 0$ as $r \rightarrow 0$ or $r \rightarrow \infty$.

(2) For $L$ is elliptic away from the $z$-axis, from elliptic theory, we know $\psi=$ $\int G_{\varsigma} d \mu$ is $C^{\prime}$ on $r>0$ for $\varsigma \in L^{\infty}$. Notice the function $\psi_{n} / r^{2}$ is $C^{\prime}$ on $r \geq 0$, where $\psi_{n}$ is introduced in Appendix A, with $-L \psi_{n} / r^{2}=\zeta_{n} \in L^{\infty}$. Now, define $\phi(r, z)=\psi(r, z) / r^{2}$ on $r>0$ for $\psi=\int G \zeta d \mu$.

PROPOSITION 2. (a)

$$
|\phi(r, z)| \leq C|\varsigma|_{\infty}^{3 / 5} P(|\zeta|)^{2 / 5}
$$

(b)

for $r^{2}+z^{2} \leq 3$.

$$
\left|\phi_{r}(r, z)\right|,\left|\phi_{z}(r, z)\right| \leq C\left(|\zeta|_{\infty}^{3 / 5} P(|\varsigma|)^{2 / 5}+|\zeta|_{\infty}^{5 / 6} P(|\zeta|)^{1 / 6}\right)
$$

Here, we follow some ideas in $[\mathbf{1 7}, \mathbf{1}]$. Consider $\phi, \zeta$ as functions on $R^{5}$ via $r^{2}=x_{1}^{2}+\cdots+x_{4}^{2}, z=x_{5}$. One can readily see $-\varsigma=\Delta_{5} \phi, \phi=\int G_{5} \varsigma d x, \int|\varsigma| d x=$ $\left(A_{4} / 2 \pi\right) P(|\varsigma|)$. Here, $\Delta_{5}=$ the Laplace operator on $R^{5}, G_{5}=1 / 3 A_{5}\left|x-x^{\prime}\right|^{3}$, with $A_{4}\left(A_{5}\right)=$ the area of unit sphere in $R^{4}\left(R^{5}\right)$.

PROOF OF PROPOSITION 2. (a) To each $x \in R^{5}$,

$$
\begin{aligned}
|\phi(x)| & \leq \int_{\left|x-x^{\prime}\right|<\varepsilon}\left|G_{5} \zeta\right| d x^{\prime}+\int_{\left|x-x^{\prime}\right| \geq \varepsilon}\left|G_{5}\right| d x^{\prime} \\
& \leq \frac{C}{2}\left[|\zeta|_{\infty} \varepsilon^{2}+\frac{1}{\varepsilon^{3}} \int|\zeta| d x\right]=C|\zeta|_{\infty}^{3 / 5}\left(\int|\zeta| d x\right)^{2 / 5}
\end{aligned}
$$

by choosing $\varepsilon^{5}=\int|\varsigma| d x /|\varsigma|_{\infty}$. 
Thus, (a) follows by observing $\int|\zeta| d x=\left(A_{4} / 2 \pi\right) P(|\zeta|)$.

(b) Denote by $\|u\|_{m, p, \Omega}=\sum_{|\alpha| \leq m}\left(\int_{\Omega}\left|D^{\alpha} u\right|^{p} d x\right)^{1 / p}$ the Sobolev norm of $u$ on the domain $\Omega$. An interior estimate for Laplace operator $A_{5}$ gives

$$
\|\phi\|_{2, P, \Omega^{\prime}} \leq C\left(\|\zeta\|_{0, p, \Omega}+\|\phi\|_{0, p, \Omega}\right)
$$

where $\Omega^{\prime}=\left\{x \in R^{5} \mid r^{2}+z^{2} \leq 3\right\} . \Omega=\left\{x \in R^{5} \mid r^{2}+z^{2} \leq 4\right\}$.

From (a),

$$
\|\phi\|_{0, p, \Omega} \leq C|\zeta|_{\infty}^{3 / 5}\left(\int|\zeta| d x\right)^{2 / 5}
$$

Notice also,

$$
\|\zeta\|_{0, p, \Omega} \leq C|\zeta|_{\infty}^{(p-1) / p}\left(\int|\zeta| d x\right)^{1 / p} .
$$

Combining inequalities (B.1), (B.2), (B.3), one gets

$$
\|\phi\|_{2, p, \Omega^{\prime}} \leq C\left[|\zeta|_{\infty}^{3 / 5} P(|\zeta|)^{2 / 5}+|\zeta|_{\infty}^{(p-1) / p} P(|\zeta|)^{1 / p}\right]
$$

Thus,

$$
\left|\phi_{r}\right|,\left|\phi_{z}\right| \leq C\left[|\zeta|_{\infty}^{3 / 5} P(|\zeta|)^{2 / 5}+|\zeta|_{\infty}^{5 / 6} P(|\zeta|)^{1 / 6}\right] \quad \text { for } r^{2}+z^{2} \leq 3
$$

by using Sobolev inequality with $p=6$. Hence, we complete the proof of Proposition 2 .

C. A geometric consideration about $\partial H_{\varepsilon}$. For small $\varepsilon>0, H_{\varepsilon}$ is a convex set with $C^{1}$ boundary $\partial H_{\varepsilon}$. Here, we give some information about variations of tangent lines along $\partial H_{\varepsilon}$.

PROPOSITION 3. There exists $\delta>0$ such that, for sufficiently small $\varepsilon>0$, the difference between the angles of tangent lines with the $z$-axis of two points $(r, z)$, $\left(r^{\prime}, z^{\prime}\right)$ on $\partial H_{\varepsilon}$ is smaller than $\pi / 2-\delta$, when $s \leq r / 2 .\left(\right.$ Recall $S^{2}=\left(r-r^{\prime}\right)^{2}+$ $\left(z-z^{\prime}\right)^{2}$.)

ProOF. Take $m=1 / 3, r^{*}=m / 2 \sqrt{1+m^{2}}, z^{*}=1 / 2$. It suffices to show that the proposition is valid for $0<r<r^{*}, z>z^{*}$. Define a sequence of points $P_{i}=\left(r\left(P_{i}\right), z\left(P_{i}\right)\right), i=1,2,3,4$, counterclockwise on $\partial H_{\varepsilon}$ with $z\left(P_{i}\right) \geq 0$ via the following characterizations:

at $P_{1}, z\left(P_{1}\right)=0, r\left(P_{1}\right)$ is small,

at $P_{2}$, the tangent line of $\partial H_{\varepsilon}$ has slope $m$,

at $P_{3}$, the tangent line of $\partial H_{\varepsilon}$ has slope $1 / m$,

at $P_{4}, r\left(P_{4}\right)=3 m / 4 \sqrt{1+m^{2}}$ (so that its tangent line has a slope that is close to $\left.-\sqrt{16+7 m^{2}} / 3 m<-1 / m\right)$.

Thus, the variation of angles along the $\operatorname{arc} P_{2} P_{4}$ or the $\operatorname{arc} P_{1} P_{3}$ on $\partial H_{\varepsilon}$ is less than $\pi / 2-\delta$ for some $\delta>0$. Computations show that

$$
r\left(P_{2}\right)=(10 m \gamma(\varepsilon))^{1 / 3}(1+o(1)), \quad r\left(P_{3}\right)=(10 \gamma(\varepsilon) / m)^{1 / 3}(1+o(1)) .
$$

As $m^{2 / 3}<1 / 2$, the two points $(r, z),\left(r^{\prime}, z^{\prime}\right)$ with $s \leq r / 2$ lie in either the arc $P_{2} P_{4}$ or $P_{1} P_{3}$. Thus our proposition follows. 
COROLlaRY 2. $\left|d s / d \alpha^{\prime}\right| \geq \frac{1}{3} \cos (\pi / 2-\delta)$ on $\partial H_{\varepsilon}$ if $s \leq r / 2$.

ProOF. We parametrize $X \in \partial H_{\varepsilon}$ by its $\alpha$-coordinate as $X\left(\alpha^{\prime}\right)=\left(\frac{1}{2}, 0\right)+$ $\left(\rho_{0} \cos \alpha^{\prime}, \rho_{0} \sin \alpha^{\prime}\right)$, where $\rho_{0}=$ the Euclidean distant between $\left(\frac{1}{2}, 0\right)$ and $X$. Let $(r, z)=X(\alpha) \in \partial H_{\varepsilon}$. Thus,

$$
\frac{d s}{d \alpha^{\prime}}=\frac{\left[X\left(\alpha^{\prime}\right)-X(\alpha)\right]}{s} \cdot \frac{d X}{d \alpha^{\prime}}=\left|\frac{d X}{d \alpha^{\prime}}\right| \cos \eta
$$

where $\eta$ is the angle between $X\left(\alpha^{\prime}\right)-X(\alpha)$ and $d X / d \alpha^{\prime}\left(\alpha^{\prime}\right)$. By a mean-value theorem, $\eta$ equals to the angle between $d X / d \alpha^{\prime}\left(\alpha^{\prime \prime}\right)$ and $d X / d \alpha^{\prime}\left(\alpha^{\prime}\right)$ for some $\alpha^{\prime \prime}$ between $\alpha$ and $\alpha^{\prime}$. So, $\eta \leq \pi / 2-\delta$ by the above proposition. Notice also $\left|d X / d \alpha^{\prime}\right| \geq$ $\rho_{0} \geq \frac{1}{3}$. Hence, $\left|d s / d \alpha^{\prime}\right| \geq \frac{1}{3} \cos (\pi / 2-\delta)$.

\section{REFERENCES}

1. C. J. Amick and L. E. Fraenkel, The uniqueness of Hill's spherical vortex, Arch. Rational Mech. Anal. 92 (1986), 91-119.

2. V. I. Arnold, Conditions for nonlinear stability of stationary plane curvilinear flows of an ideal fluid, Soviet Math. Dokl. 6 (1965), 773-777.

3. 79 (1969), 267-269.

4. T. B. Benjamin, The stability of solitary waves, Proc. Roy. Soc. London 328 (1972), 153-183.

5. - The alliance of practical and analytic insights into the nonlinear problems of fluid mechanics, Applications of Methods of Functional Analysis to Problems of Mechanics, Lecture Notes in Math., vol. 503, Springer-Verlag, Berlin and New York, 1976, pp. 8-29.

6. __ Impulse, flow force, and variational principles, IMA J. Appl. Math. 32 (1984), 3-68.

7. G. S. Deem and N. J. Zabusky, Vortex waves: Stationary 'V states', interactions, recurrence, and breaking, Phys. Rev. Lett. 40 (1978), 859-862.

8. L. E. Fraenkel, Examples of steady vortex rings of small cross-section in an ideal fluid, J. Fluid Mech. 51 (1972), 119-135.

9. L. E. Fraenkel and M. S. Berger, A global theory of steady vortex rings in an ideal fluid, Acta Math. 132 (1974), 14-51.

10. A. Friedman and B. Turkington, Vortex rings: Existence and asymptotic estimates, Trans. Amer. Math. Soc. 268 (1981), 1-37.

11. M. J. M. Hill, On a spherical vortex, Philos. Trans. Roy. Soc. London Ser. A 185 (1894), 213-245.

12. D. Holm, J. Marsden, T. Ratiu, and A. Weinstein, Nonlinear stability of fluid and plasma equilibria, Phys. Rep. 123 (1985), 1-116.

13. B. Kelvin, On the stability of steady and of periodic fluid motion, Philos. Mag. 22 (1887), 459-464, 529-539.

14. H. Lamb, Hydrodynamics, Dover, New York, 1945.

15. J. Marsden and A. Weinstein, Coadjoint orbits, vortices, and Clebsch variables for incompressible fluids, Physica D 7 (1983), 305-323.

16. H. K. Moffatt and D. W. Moore, The response of Hill's spherical vortex to a small axisymmetric disturbance, J. Fluid Mech. 73 (1978),

17. W.-M. Ni, On the existence of global vortex rings, J. Analyse Math. 37 (1980), 208-247.

18. J. Norbury, A steady vortex ring close to Hill spherical vortex. Proc. Cambridge Philos. Soc. 72 (1972), 253-284.

19. _ A family of steady rings, J. Fluid Mech. 57 (1973), 417-431.

20. Y. Tang, Nonlinear stability of vortex patches, Thesis, State Univ. of New York at Buffalo, 1985.

21. Y.-H. Wan and M. Pulvirenti, Nonlinear stability of circular vortex patches, Comm. Math. Phys. 99 (1985), 435-450.

Department of Mathematics, State University of New York at Buffalo, NEW YORK 14214 